\title{
Heterosis, Heritability and Combining Abilities for some Earliness Traits in Squash (Cucurbita pepo, L.) \\ El-Adl, A. M..; ${ }^{1}$ A. H. Abd El-Hadi; ${ }^{1}$ Horeya M. Fathy and M. A. Abdein ${ }^{2}$
}

\begin{abstract}
This study was conducted at Kaha Research Farm of Vegetables Breeding Department, Horticultural Research Institute, (HRI), Agric. Res. Center (ARC), Giza, Egypt, during the period from 2008 to 2010 . The study aimed to estimate heterosis versus the mid-parents and the better parent, general (GCA) and specific (SCA) combining ability variances, nature of gene action, heritability in both broad and narrow senses in some summer squash for economic earliness traits. These traits included number of first female flowering node (No.1 ${ }^{\text {st } F . F . N) ; ~ d a t e ~ o f ~ f i r s t ~ m a l e ~}$ flower (D. ${ }^{\text {st }}$ M.F.); data of first female flower (D. ${ }^{\text {st }}$ F.F.); first picking date $\left({ }^{\text {st }}\right.$ P.D.) and early yield per plant (E.Y./P.kg.). Seven imported squash varieties, namely Eskandarani $\left(\mathbf{P}_{1}\right)$; Zucca Patisson custard white $\left(\mathbf{P}_{2}\right)$; All Green Bush $\left(P_{3}\right)$; Courgette Orelia $\left(P_{4}\right)$; Sakiz $\left(P_{5}\right)$; Copi $\left(P_{6}\right)$ and Gapla $\left(P_{7}\right)$ were used as parental varieties in the present investigation. Diallel crosses (with reciprocals) were made between these parents to obtain $42 F_{1}, 1$ hybrids according to the complete diallel crosses mating design. All genotypes were evaluated in randomized complete-blocks designs with three replications. The results indicated that the parental varieties Courgette Orelia $\left(\mathbf{P}_{4}\right)$ and Gapla $\left(\mathbf{P}_{7}\right)$ seemed to be the best combiners for earliness traits such as number of first female flowering node (No. ${ }^{\text {st }}$ F.F.N); date of first male flower (D.1 ${ }^{\text {st }}$ M.F.); data of first female flower $\left(D .1^{\text {st }}\right.$ F.F. $)$ and first picking date $\left(1^{\text {st }}\right.$ P.D. $)$. The parents Zucca Patisson custard white $\left(P_{2}\right)$ and Copi $\left(P_{6}\right)$ were the best combiners for early yield per plant (E.Y./P.kg.). In general, the performances of most $F_{1,1 r}$ hybrids were variable and the results cleared that no hybrid was the best all for earliness traits. The results showed the importance of general (GCA) and specific (SCA) combining abilities. GCA was larger than their corresponding estimates of SCA for most earliness traits. Reciprocal effects ( $r$ ) were significant for most earliness traits. In the same time, the estimates of heritability in broad sense were larger in magnitudes than their corresponding estimates of narrow sense.
\end{abstract}

Keywords: squash, earliness, heterosis, combining abilities and heritability.

\section{INTRODUCTION}

Cucurbitaceae is one of the most important botanical families for human use as favorable vegetable crop. Thus, summer squash (Cucurbita pepo, L.) is considered to be one of the most popular vegetable crops grown in Egypt. It is known as a vegetable marrow and is also called Kosa by the Egyptian. In Egypt, there are only two local cultivars of squash i.e. Balady, which is lately discarded for its prostrate growth habit and low yield and Eskandarani, which is high yielding and satisfies both the producer and consumer.

In squash and other cucurbits, $F_{1}$ hybrids were utilized aiming to increase both the productivity and earliness of traits. Many investigators studied heterosis among them; El-Adl et al., (1988) who evaluated $\mathrm{F}_{1}$ hybrids and their parents. They found that number of node to the first female flower expressed heterosis values of -1.66 and $12.48 \%$ versus the mid-parents and the better parent, respectively.

Similarly, Abd El-Maksoud et al., (2003) showed that the average means of the $F_{1}$ hybrids, $F_{1 \mathrm{r}}$ (reciprocal) hybrids and the average over all hybrids $\left(\mathrm{F}_{1,1 \mathrm{r}}\right)$ exceeded their mid-parents $\left(\mathrm{H}_{\text {M.P. }} \%\right)$ for all studied traits except for sex ratio and days to first female flower, In another study by Abd El-Hadi et al., (2005) they evaluated $12 \mathrm{~F}_{1}$ hybrids obtained from four varieties of summer squash. They reported that the estimated amounts of heterosis showed highly significant values for flowering traits. Al-Ballat (2008) found that heterosis over the mid-parents was highly significant with negative values for number of days to first female flower and number of nodes to first female flower. Jahan et al., (2012) found, in sweet gourd that node number of first female flower in the two hybrids, OP $10 \times$ OP 24 and OP $10 \times$ OP 20 recorded the highest significant positive heterosis from the mid and better parent. Tamil et al., (2012) evaluated 15 pumpkin genotypes collected from various sources. Important heterosis values were recorded for days to first female flower appearance, node number for first female flower appearance, sex ratio, days to first harvest.

Concerning, GCA and SCA variances in cucumber, Abd El-Hafez et al., (1997) evaluated a $5 \times 5$ diallel analysis to determine combining ability for some earliness traits. They reported significant positive values of SCA effects in some crosses which produced earlier flowers. El-Gendy (1999) reported that general combining ability and specific combining ability as well

\footnotetext{
${ }^{1}$ Dept. of Genetics, Faculty of Agric. Mansoura University, Egypt.

${ }^{2}$ Vegetables Breeding Department, Horticulture Res. Inst. (HRI),

ARC, Giza, Egypt.

E- mail: abdeingene@yahoo.com

Received July 22, 2014, Accepted August26, 2014
} 
as reciprocal effects were significant for days to first female flower; numbers of fruits in the first seven pickings. In summer squash, El-Sharkawy (2000) regarded that a parental inbred line $\mathrm{L}_{2}$ could be considered as a good general combiner for harvesting early yield. The results also indicated that the two crosses $\left(\mathrm{L}_{2} \times \mathrm{L}_{6}\right)$ and $\left(\mathrm{L}_{2} \times \mathrm{L}_{5}\right)$ gave the earlier $\mathrm{F}_{1}$ hybrids and these crosses possessed the highest estimates of SCA effects. In squash, Sadek (2003) illustrated that the non-additive genetic variances including dominance were the most important source of genetic variance. The results showed that both additive and non-additive genetic variances contributed in the inheritance of position of the first female flower; days to the first female flower and early yield as number and weight of fruits. Obiadalla-Ali (2006), who worked in squash and recorded data for days to first female flower and sex ratio estimated as number of female flowers/number of total flowers. The magnitude of additive genetic variance $\left(\sigma^{2} A\right)$ were positive and lower than those of non additive $\left(\sigma^{2} D\right)$ one for both traits, indicating that non additive gene action played a major role in the inheritance of both traits. Al-Araby, (2010) estimated GCA effects and indicated that the parent E-4 was the best combiner for number of days to first female flower opening; number of days to female flowering; number of nodes to first female flower and number of female flowers/plant. It also was good combiner for early number and weight of fruits/plot. Recently, Sanin et al., (2014) studied the predominance of additive gene action over the dominance type for the traits under study suggests that a recurrent selection program could serve as a strategy to increase the frequencies of genes that promote the expression of traits associated with seed production and starch content in butternut squash.

Zuradzka (1988) estimated heritability for number of female flowers in cucumber, using parental varieties, $F_{1}$ hybrids, $F_{2}$ generations and their two backcrosses for various unspecified crosses. The obtained results which showed that heritability estimates ranged from 0.38 to 0.77 in broad sense for this trait. Abd El-Hadi and ElGendy (2004) studied four squash varieties and their 12 $\mathrm{F}_{1,1 \mathrm{r}}$ hybrids. The results indicated that the magnitudes of heritability in broad sense $\left(\mathrm{h}_{\mathrm{b}}^{2} \%\right)$ were always larger than their corresponding estimates in narrow sense $\left(\mathrm{h}_{\mathrm{n}}^{2} \%\right)$ for all studied traits. Mishra et al., (2007) who worked on cucumber, stated that minimum value of heritability was observed for number of days to first female flowering.

\section{MATERIALS AND METHODS}

The genetic materials used in the present investigation included seven squash varieties belong to the species (Cucurbita pepo, L.). These parental varieties were: Eskandarani $\left(\mathrm{P}_{1}\right)$; Zucca Patisson custard white $\left(\mathrm{P}_{2}\right)$; All Green Bush $\left(\mathrm{P}_{3}\right)$; Courgette Orelia $\left(\mathrm{P}_{4}\right)$; Sakiz $\left(\mathrm{P}_{5}\right)$; Copi $\left(\mathrm{P}_{6}\right)$ and Gapla $\left(\mathrm{P}_{7}\right)$. The seeds of these parental varieties were obtained from different countries: $\left(\mathrm{P}_{1}\right)$ and $\left(\mathrm{P}_{6}\right)$ from Egypt; $\left(\mathrm{P}_{2}\right)$ from France; $\left(\mathrm{P}_{3}\right)$ from United Kingdom (U.K.); $\left(\mathrm{P}_{4}\right)$ from Germany; $\left(\mathrm{P}_{5}\right)$ from Turkey and $\left(\mathrm{P}_{7}\right)$ from Syria. The seven varieties were chosen to represent a wide rang of variation in most characters.

Plants from each parental variety were selfpollinated for three successive generations to obtain inbreds from each variety. In the summer season of 2009, all single crosses including reciprocals were made among these seven varieties according to a complete diallel crosses mating design to produce $21 \mathrm{~F}_{1}$ hybrids and their $21 \mathrm{~F}_{1 \mathrm{r}}$ (reciprocal) hybrids. In addition, the seven parental varieties were also self-pollinated to obtain enough seeds from each variety. All 49 genotypes (seven parents, $21 \mathrm{~F}_{1}$ 's and $21 \mathrm{~F}_{1 \mathrm{r}}$ reciprocals) were evaluated in a field trial in the growing summer season of 2010 at Kaha Vegetables Research Station, Kaha, Kalubia, Egypt.

The experimental design was a Randomized Complete Blocks Design (RCBD) with three replications. Data were recorded for five earliness traits. These traits were: number of first female flowering node (No. $1^{\text {st } F . F . N .) ; ~ d a t e ~ o f ~ f i r s t ~ m a l e ~ f l o w e r ~}$ (D. $1^{\text {st }}$ M.F.); data of first female flower (D. ${ }^{\text {st }}$ F.F.); first

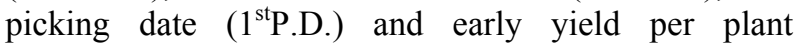
(E.Y./P.kg.).

Differences among genotypic means for all earliness traits were tested for significance according to the Ftest. The form of analysis of variance and the expectations of mean squares were as outlined by Steel and Torrie (1960).

The amounts of heterosis were determined as the percentage deviation from the means of the $F_{1}$ hybrids $\left(F_{1}\right), F_{1 r}$ reciprocal hybrids $\left(F_{1 r}\right)$ and over all $F_{1,1 r}$ hybrids from the average of all parents (mid-parents) or the better parent.

In this investigation, seven parental varieties were utilized in a complete diallel crosses mating design to estimate general combining ability (GCA) and specific combining ability (SCA). In addition, the variances of reciprocal effect (r) could be also obtained. The procedures of this analysis were described by Griffing (1956) method I. The estimates of GCA variance $\left(\delta^{2} \mathrm{~g}\right)$ and SCA variance $\left(\delta^{2} \mathrm{~s}\right)$ could be expressed in terms of genetic variances according to Matzingar \& Kempthorne (1956) and Cockerham (1963).

\section{RESULTS AND DISCUSSIONS}


Squash varieties possess a wide range of variation for earliness traits. Vegetable breeders usually use this variability as a tool to improve squash varieties through selection programs or to produce $\mathrm{F}_{1}$ hybrids to make use of hybrid vigor phenomena and to obtain early yielding hybrids.

\section{Analysis of variance:-}

The analysis of variance and the mean squares for earliness traits for all genotypes were made and the results are presented in Table 1 .

The mean squares of genotypes for the five earliness traits; number of first female flowering node (No. ${ }^{\text {st }}$ F.F.N.); date of first male flower (D. $1^{\text {st }}$ M.F.); data of first female flower (D. ${ }^{\text {st }}$ F.F.); first picking date ( 1 st P.D.) and early yield per plant (E.Y./P.kg.) were highly significant. Therefore, this result reflected the presence of real difference among them. This finding was true for all other studies. The significance of mean squares of genotypes suggested that the planned comparisons to understanding the nature of variation and the determination of the amount of heterosis for these traits were valid and therefore, could be made. Similar results were obtained by El-Sharkawy (2000); Al-Ballat (2008); Al-Araby, (2010); Jahan et al., (2012); Tamil et al., (2012) and Sanin et al., (2014).

\section{The mean performance of all genotypes:}

The mean performances of parental varieties and their $F_{1}$ hybrids including reciprocals for earliness traits are presented in Tables 2, 3 and 4.

The means of the seven parental varieties showed that the lowest parent is $\mathrm{P}_{4}$ for earliness trait No. $1^{\text {st }}$ F.F.N. (desirable). While, the highest parent for all studied earliness traits was $\mathrm{P}_{6}$ (undesirable) except E.Y./P.kg. (desirable). On the other hand, the parental variety $\mathrm{P}_{7}$ was the lowest parent for D. $1^{\text {st }}$ M.F.; D. $1^{\text {st }}$ F.F. and $1^{\text {st }}$ P.D., respectively (desirable). The results revealed that the E.Y./P.kg. ranged from 0.841 to 1.175 $\mathrm{kg} /$ Plant for $\mathrm{P}_{3}$ and $\mathrm{P}_{6}$ parental varieties, respectively.

The results indicated that parental variety $\mathrm{P}_{7}$ started to flower earlier than the other studied varieties. This finding would be confirmed by the means of days to first male flower (D. $1^{\text {st }}$ M.F.) 35.13 days; the days of first female flower (D. ${ }^{\text {st }}$ F.F.), 32.83 days and first picking date ( $1^{\text {st }}$ P.D.) 34.93 days. Furthermore, the results indicated that $\mathrm{P}_{6}$ was a very late variety. It is also regarded from the results that the parental variety $\mathrm{P}_{7}$ was the earlier parent (desirable) and has the lowest parent for $1^{\text {st }}$ P.D.

In addition, the earlier $F_{1}$ hybrids and $F_{1 r}$ (reciprocal) hybrids were obtained when the hybridization included any one of the earlier parental variety or both the two varieties $\mathrm{P}_{7}$ and $\mathrm{P}_{4}$ with respect to all the studied earliness traits. For instance, the hybrid $\mathrm{P}_{3} \times \mathrm{P}_{4}$ exhibited the lowest mean value (desirable) for nodes to the first female flower (No. $1^{\text {st }}$ F.F.N.), but the hybrid $\mathrm{P}_{1} \times \mathrm{P}_{6}$ exhibited the highest mean value (undesirable) for nodes to the first female flower (No. $1^{\text {st } F . F . N .) . ~ O n ~ t h e ~ o t h e r ~ h a n d, ~ t h e ~} F_{1 r}$ (reciprocal) hybrid $\mathrm{P}_{7} \times \mathrm{P}_{4}$ was the lowest (desirable) and the hybrid $\mathrm{P}_{6} \times \mathrm{P}_{1}$ was the highest (undesirable) for the same trait.

The results indicated that the highest (undesirable) $\mathrm{F}_{1}$ hybrid for D. ${ }^{\text {st }}$ F.F. was $\mathrm{P}_{1} \times \mathrm{P}_{6}$ with the mean value of 34.07 days. Whereas, the highest $\mathrm{F}_{1 \mathrm{r}}$ (reciprocal) hybrid for D. ${ }^{\text {st }}$ F.F. was $\mathrm{P}_{6} \times \mathrm{P}_{2}$ with the mean value of 34.93 days. On the other hand, the $\mathrm{F}_{1}$ hybrid $\mathrm{P}_{2} \times \mathrm{P}_{4}$ was the lowest (desirable) with the mean value of 26.87 days. While, $\mathrm{F}_{1 \mathrm{r}}$ (reciprocal) hybrid $\mathrm{P}_{7} \times \mathrm{P}_{4}$ was the lowest (desirable) with the mean value 26.37 of days for the same trait.

Table 1. Analysis of variance and expectation of mean squares for earliness traits

\begin{tabular}{|c|c|c|c|c|c|c|}
\hline \multirow[b]{2}{*}{ S.O.V. } & \multirow[b]{2}{*}{ d.f } & \multicolumn{5}{|c|}{ Earliness traits } \\
\hline & & No.1 ${ }^{\text {st }}$ F.F.N. & D.1 ${ }^{\text {st }}$ M.F. & D.1 ${ }^{\text {st F.F. }}$ & 1 ${ }^{\text {st P.D. }}$ & E.Y./P.kg. \\
\hline Reps & 2 & 0.012 & $6.740 * *$ & 0.005 & 0.149 & 0.002 \\
\hline Genotypes & 48 & $1.278 * *$ & $14.486^{* *}$ & $40.086^{* *}$ & $39.048 * *$ & $0.387 * *$ \\
\hline Error & 96 & 0.245 & 0.800 & 0.626 & 0.631 & 0.003 \\
\hline
\end{tabular}

*,** Significant at 0.05 and 0.01 levels of probability, respectively.

Table 2. The mean performances of the seven parental varieties for earliness traits

\begin{tabular}{|c|c|c|c|c|c|c|}
\hline \multirow[b]{2}{*}{ No. } & \multirow{2}{*}{$\begin{array}{l}\text { Parental } \\
\text { varieties }\end{array}$} & \multicolumn{5}{|c|}{ Earliness traits } \\
\hline & & No.1 ${ }^{\text {st F.F.N. }}$ & D.1 ${ }^{\text {st M.F. }}$ & D.1 ${ }^{\text {st }}$ F.F. & $\mathbf{1}^{\text {st }}$ P.D. & E.Y./P.kg. \\
\hline 1 & $\mathrm{P}_{1}$ & 5.37 & 39.93 & 41.23 & 43.37 & 1.056 \\
\hline 2 & $\mathrm{P}_{2}$ & 5.43 & 39.63 & 38.07 & 40.87 & 1.139 \\
\hline 3 & $\mathrm{P}_{3}$ & 4.91 & 38.07 & 36.17 & 38.27 & $0.841^{\mathrm{L}}$ \\
\hline 4 & $\mathrm{P}_{4}$ & $4.11^{L}$ & 36.17 & 34.87 & 36.87 & 0.885 \\
\hline 5 & $\mathrm{P}_{5}$ & 4.93 & 37.13 & 35.23 & 37.33 & 1.031 \\
\hline 6 & $\mathrm{P}_{6}$ & $6.09^{\mathrm{H}}$ & $43.33^{\mathrm{H}}$ & $44.17^{\mathrm{H}}$ & $46.07^{\mathrm{H}}$ & $1.175^{\mathrm{H}}$ \\
\hline 7 & $\mathrm{P}_{7}$ & 4.41 & $35.13^{\mathrm{L}}$ & $32.83^{\mathrm{L}}$ & $34.93^{\mathrm{L}}$ & 1.044 \\
\hline $\mathrm{L} . \mathrm{S}$ & & 0.80 & 1.44 & 1.27 & 1.28 & 0.08 \\
\hline L.S & & 1.05 & 1.91 & 1.69 & 1.69 & 0.11 \\
\hline
\end{tabular}


$\mathrm{H}=$ The highest value

$\mathrm{L}=$ The lowest value

Table 3. The mean performances of the $21 \mathrm{~F}_{1}$ hybrids for earliness traits

\begin{tabular}{|c|c|c|c|c|c|c|}
\hline \multirow[b]{2}{*}{ No } & \multirow[b]{2}{*}{$F_{1}$ hybrids } & \multicolumn{5}{|c|}{ Earliness traits } \\
\hline & & No. ${ }^{\text {st }}$ F.F.N. & D.1 ${ }^{\text {st }}$ M.F. & D. ${ }^{\text {st } F . F . ~}$ & $1^{\text {st P.D. }}$ & E.Y./P.kg. \\
\hline 1 & $\mathrm{P}_{1} \times \mathrm{P}_{2}$ & 4.02 & 32.27 & 28.07 & 31.17 & 1.797 \\
\hline 2 & $\mathrm{P}_{1} \times \mathrm{P}_{3}$ & 3.94 & 32.63 & 30.23 & 32.27 & 1.086 \\
\hline 3 & $\mathrm{P}_{1} \times \mathrm{P}_{4}$ & 3.18 & $30.67^{\mathrm{L}}$ & 27.67 & 29.83 & 1.104 \\
\hline 4 & $\mathrm{P}_{1} \times \mathrm{P}_{5}$ & 3.72 & 33.17 & 29.23 & 31.27 & 1.089 \\
\hline 5 & $\mathrm{P}_{1} \times \mathrm{P}_{6}$ & $4.81^{\mathrm{H}}$ & 35.77 & $34.07^{\mathrm{H}}$ & $36.23^{\mathrm{H}}$ & 1.904 \\
\hline 6 & $\mathrm{P}_{1} \times \mathrm{P}_{7}$ & 3.84 & 33.13 & 28.73 & 30.73 & 1.091 \\
\hline 7 & $\mathrm{P}_{2} \times \mathrm{P}_{3}$ & 4.24 & 34.67 & 29.03 & 31.67 & 1.919 \\
\hline 8 & $\mathrm{P}_{2} \times \mathrm{P}_{4}$ & 3.51 & 32.23 & $26.87^{\mathrm{L}}$ & 29.77 & 1.587 \\
\hline 9 & $\mathrm{P}_{2} \times \mathrm{P}_{5}$ & 3.93 & 33.93 & 27.73 & 30.77 & 1.689 \\
\hline 10 & $\mathrm{P}_{2} \times \mathrm{P}_{6}$ & 4.18 & 34.97 & 32.77 & 35.67 & $2.053^{\mathrm{H}}$ \\
\hline 11 & $\mathrm{P}_{2} \times \mathrm{P}_{7}$ & 3.39 & 33.67 & 28.87 & 31.77 & 1.542 \\
\hline 12 & $\mathrm{P}_{3} \times \mathrm{P}_{4}$ & $3.12^{\mathrm{L}}$ & 32.87 & 28.97 & 30.87 & $1.051^{\mathrm{L}}$ \\
\hline 13 & $\mathrm{P}_{3} \times \mathrm{P}_{5}$ & 3.77 & 34.23 & 29.57 & 31.83 & 1.113 \\
\hline 14 & $\mathrm{P}_{3} \times \mathrm{P}_{6}$ & 4.09 & 35.93 & 31.93 & 34.13 & 1.776 \\
\hline 15 & $\mathrm{P}_{3} \times \mathrm{P}_{7}$ & 3.34 & 34.73 & 29.13 & 30.83 & 1.126 \\
\hline 16 & $\mathrm{P}_{4} \times \mathrm{P}_{5}$ & 3.28 & 33.63 & 30.83 & 32.93 & 1.131 \\
\hline 17 & $\mathrm{P}_{4} \times \mathrm{P}_{6}$ & 3.59 & 35.73 & 31.73 & 34.07 & 1.218 \\
\hline 18 & $\mathrm{P}_{4} \times \mathrm{P}_{7}$ & 3.49 & 34.93 & 26.93 & $28.73^{\mathrm{L}}$ & 1.074 \\
\hline 19 & $\mathrm{P}_{5} \times \mathrm{P}_{6}$ & 4.58 & $36.23^{\mathrm{H}}$ & 33.93 & 36.17 & 1.389 \\
\hline 20 & $\mathrm{P}_{5} \times \mathrm{P}_{7}$ & 3.99 & 34.47 & 31.67 & 33.77 & 1.068 \\
\hline 21 & $\mathrm{P}_{6} \times \mathrm{P}_{7}$ & 4.39 & 32.83 & 31.27 & 33.37 & 1.338 \\
\hline L.S.I & & 0.80 & 1.44 & 1.27 & 1.28 & 0.08 \\
\hline L.S.I & & 1.05 & 1.91 & 1.69 & 1.69 & 0.11 \\
\hline
\end{tabular}

Table 4.The mean performances of the $F_{1 \mathrm{r}}$ hybrids for earliness traits

\begin{tabular}{|c|c|c|c|c|c|c|}
\hline \multirow[b]{2}{*}{ No } & \multirow[b]{2}{*}{$F_{1 \mathbf{r}}$ hybrids } & \multicolumn{5}{|c|}{ Earliness traits } \\
\hline & & No.1 ${ }^{\text {st }}$ F.F.N. & D.1 ${ }^{\text {st }}$ M.F. & D.1 ${ }^{\text {st F.F. }}$ & 1 $^{\text {st P.D. }}$ & E.Y./P.kg. \\
\hline 22 & $\mathrm{P}_{2} \times \mathrm{P}_{1}$ & 3.78 & 34.57 & 29.83 & 32.83 & 1.734 \\
\hline 23 & $\mathrm{P}_{3} \times \mathrm{P}_{1}$ & 3.32 & 34.87 & 31.77 & 33.87 & 1.142 \\
\hline 24 & $\mathrm{P}_{3} \times \mathrm{P}_{2}$ & 4.23 & 33.57 & 28.77 & 31.93 & 1.875 \\
\hline 25 & $\mathrm{P}_{4} \times \mathrm{P}_{1}$ & 3.69 & 33.23 & 27.77 & 29.97 & 1.122 \\
\hline 26 & $\mathrm{P}_{4} \times \mathrm{P}_{2}$ & 3.43 & 34.13 & 28.17 & 31.37 & 1.644 \\
\hline 27 & $\mathrm{P}_{4} \times \mathrm{P}_{3}$ & 3.27 & 34.83 & 27.37 & 29.33 & $1.047^{\mathrm{L}}$ \\
\hline 28 & $\mathrm{P}_{5} \times \mathrm{P}_{1}$ & 3.97 & 33.87 & 28.83 & 31.13 & 1.119 \\
\hline 29 & $\mathrm{P}_{5} \times \mathrm{P}_{2}$ & 4.19 & 35.07 & 30.77 & 33.97 & 1.932 \\
\hline 30 & $\mathrm{P}_{5} \times \mathrm{P}_{3}$ & 3.16 & 34.77 & 28.67 & 31.07 & 1.161 \\
\hline 31 & $\mathrm{P}_{5} \times \mathrm{P}_{4}$ & 3.79 & 34.33 & 29.07 & 31.23 & 1.136 \\
\hline 32 & $\mathrm{P}_{6} \times \mathrm{P}_{1}$ & $4.89^{\mathrm{H}}$ & 36.13 & 34.67 & 36.93 & 1.731 \\
\hline 33 & $\mathrm{P}_{6} \times \mathrm{P}_{2}$ & 4.82 & $36.73^{\mathrm{H}}$ & $34.93^{\mathrm{H}}$ & $37.83^{\mathrm{H}}$ & 1.896 \\
\hline 34 & $\mathrm{P}_{6} \times \mathrm{P}_{3}$ & 4.31 & 36.27 & 33.17 & 35.27 & 1.356 \\
\hline 35 & $\mathrm{P}_{6} \times \mathrm{P}_{4}$ & 3.66 & 35.23 & 31.87 & 33.83 & 1.947 \\
\hline 36 & $\mathrm{P}_{6} \times \mathrm{P}_{5}$ & 4.71 & 35.63 & 34.33 & 36.27 & 1.228 \\
\hline 37 & $\mathrm{P}_{7} \times \mathrm{P}_{1}$ & 3.68 & 32.77 & 29.37 & 31.47 & 1.083 \\
\hline 38 & $\mathrm{P}_{7} \times \mathrm{P}_{2}$ & 4.04 & 34.17 & 27.87 & 30.67 & $2.031^{\mathrm{H}}$ \\
\hline 39 & $\mathrm{P}_{7} \times \mathrm{P}_{3}$ & 3.91 & 33.07 & 29.17 & 31.33 & 1.071 \\
\hline 40 & $\mathrm{P}_{7} \times \mathrm{P}_{4}$ & $3.06^{\mathrm{L}}$ & $31.97^{\mathrm{L}}$ & $26.37^{\mathrm{L}}$ & $28.17^{\mathrm{L}}$ & 1.059 \\
\hline 41 & $\mathrm{P}_{7} \times \mathrm{P}_{5}$ & 3.88 & 32.93 & 29.67 & 31.87 & 1.149 \\
\hline 42 & $\mathrm{P}_{7} \times \mathrm{P}_{6}$ & 4.12 & 33.77 & 31.43 & 33.33 & 1.839 \\
\hline L.S & & 0.80 & 1.44 & 1.27 & 1.28 & 0.08 \\
\hline L.S. & & 1.05 & 1.91 & 1.69 & 1.69 & 0.11 \\
\hline
\end{tabular}


$\mathrm{H}=$ The highest value

\section{Heterosis:-}

3.1. Heterosis versus the mid-parents $\left(\mathrm{H}_{\mathrm{M.P.}} \%\right)$ :-

Heterosis versus the mid-parents $\left(\mathrm{H}_{\text {M.P. } \%)}\right.$ was estimated for earliness traits and the results are presented in Tables 5 and 6 .

The heterosis percentage of the $42 \mathrm{~F}_{1,1 \mathrm{r}}$ hybrids relative to mid-parents for earliness traits. All $F_{1,1 \mathrm{r}}$ hybrids showed highly significant and (desirable) negative heterotic values for No. $1^{\text {st }}$ F.F.N; D. $1^{\text {st }}$ M.F; D. ${ }^{\text {st }}$ F.F. and $1{ }^{\text {st }}$ P.D. toward earliness.

The results showed that heterosis percentages relative to mid-parents were found to range from -50.21 to $-14.49 \%$ for No. $1^{\text {st } F . F . N . ; ~}-19.28$ to $-2.11 \%$ for D. $1^{\text {st }}$ M.F; -29.10 to $-6.67 \%$ for D. ${ }^{\text {st F.F }}-26.00$ to -

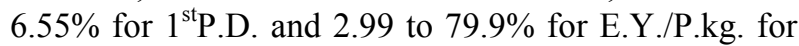
$\mathrm{F}_{1}$ hybrids. While, the values ranged for from -36.60 to $-14.83 \% ;-13.04$ to $-5.97 \% ;-26.99$ to $-12.83 \% ;-25.44$ to $-11.81 \%$ and 3.13 to $93.3 \%$ for No. $1^{\text {st } F . F . N . ; ~}$
D. ${ }^{\text {st }}$ M.F.; D. $1^{\text {st } F . F . ; ~} 1^{\text {st }}$ P.D and E.Y./P.kg., respectively $\mathrm{F}_{1 \mathrm{r}}$ (reciprocal) hybrids.

On the other hand, $18 \mathrm{~F}_{1}$ and $19 \mathrm{~F}_{1 \mathrm{r}}$ hybrids out of 21 $F_{1}$ and $21 F_{1 r}$ hybrids showed highly significant and positive (desirable) values for mid-parents heterosis estimates for E.Y./P.kg., respectively.

Concerning earliness traits for heterosis versus the mid-parents all the $21 \mathrm{~F}_{1}$ hybrids had negative and highly significant (desirable) values for most studied earliness traits except the $\mathrm{F}_{1}$ hybrid $\mathrm{P}_{5} \times \mathrm{P}_{7}$ which showed insignificant values for E.Y./P.kg. In the same time all the $21 \mathrm{~F}_{1 \mathrm{r}}$ hybrids showed highly significant and negative (desirable) estimates for most studied earliness traits.

These results were in agreement with the results obtained by El-Adl et al., (1988); Abd El-Maksoud et al., (2003); Abd El-Hadi et al., (2005); Obiadalla-Ali (2006); Al-Ballat (2008); Al-Araby (2010); Jahan et al., (2012) and Tamil, et al., (2012).

Table 5. Heterosis versus the mid-parents of the $F_{1}$ hybrids for earliness traits

\begin{tabular}{|c|c|c|c|c|c|c|}
\hline \multirow[b]{2}{*}{ No. } & \multirow{2}{*}{$\begin{array}{c}F_{1} \\
\text { hybrids }\end{array}$} & \multicolumn{5}{|c|}{ Earliness traits } \\
\hline & & No. ${ }^{\text {st }}$ F.F.N. & D. $1^{\text {st }}$ M.F. & D. $1^{\text {st }}$ F.F. & $\mathbf{1}^{\text {st }}$ P.D. & E.Y./P.kg. \\
\hline 1 & $\mathrm{P}_{1} \times \mathrm{P}_{2}$ & $-28.61 * *$ & $-18.83^{* *}$ & $-29.10 * * \mathrm{~L}$ & $-26.00 * * \mathrm{~L}$ & $66.7^{* *}$ \\
\hline 2 & $\mathrm{P}_{1} \times \mathrm{P}_{3}$ & $-50.21 * * \mathrm{~L}$ & $-16.29 * *$ & $-21.84 * *$ & $-20.87 * *$ & $14.5 * *$ \\
\hline 3 & $\mathrm{P}_{1} \times \mathrm{P}_{4}$ & $-32.37 * *$ & $-19.28 * * \mathrm{~L}$ & $-27.08 * *$ & $-25.44 * *$ & $13.8^{* *}$ \\
\hline 4 & $\mathrm{P}_{1} \times \mathrm{P}_{5}$ & $-27.87 * *$ & $-13.77 * *$ & $-23.51 * *$ & $-22.48 * *$ & 4.43 \\
\hline 5 & $\mathrm{P}_{1} \times \mathrm{P}_{6}$ & $-14.96 * *$ & $-13.94 * *$ & $-20.19 * *$ & $-18.94 * *$ & $70.7 * *$ \\
\hline 6 & $\mathrm{P}_{1} \times \mathrm{P}_{7}$ & $-22.34 * *$ & $-11.61 * *$ & $-22.38 * *$ & $-21.29 * *$ & 3.79 \\
\hline 7 & $\mathrm{P}_{2} \times \mathrm{P}_{3}$ & $-23.08 * *$ & $-10.39 * *$ & $-21.69 * *$ & $-20.03 * *$ & $97.9 * * \mathrm{H}$ \\
\hline 8 & $\mathrm{P}_{2} \times \mathrm{P}_{4}$ & $-30.64 * *$ & $-14.91 * *$ & $-26.23 * *$ & $-23.45 * *$ & $59.9 * *$ \\
\hline 9 & $\mathrm{P}_{2} \times \mathrm{P}_{5}$ & $-28.40 * *$ & $-11.60 * *$ & $-24.33 * *$ & $-21.26 * *$ & $58.6 * *$ \\
\hline 10 & $\mathrm{P}_{2} \times \mathrm{P}_{6}$ & $-31.11 * *$ & $-15.96 * *$ & $-20.23 * *$ & $-17.90 * *$ & $80.5 * *$ \\
\hline 11 & $\mathrm{P}_{2} \times \mathrm{P}_{7}$ & $-34.84 * *$ & $-11.25 * *$ & $-18.48 * *$ & $-16.22 * *$ & $43.8 * *$ \\
\hline 12 & $\mathrm{P}_{3} \times \mathrm{P}_{4}$ & $-31.06 * *$ & $-11.45 * *$ & $-18.16^{* *}$ & $-17.78 * *$ & $21.7 * *$ \\
\hline 13 & $\mathrm{P}_{3} \times \mathrm{P}_{5}$ & $-23.02 * *$ & $-9.27 * *$ & $-16.90 * *$ & $-15.82 * *$ & $19.1 * *$ \\
\hline 14 & $\mathrm{P}_{3} \times \mathrm{P}_{6}$ & $-25.99 * *$ & $-11.76^{* *}$ & $-20.50 * *$ & $-19.08 * *$ & $76.3 * *$ \\
\hline 15 & $\mathrm{P}_{3} \times \mathrm{P}_{7}$ & $-29.24 * *$ & $-5.01 * *$ & $-15.65^{* *}$ & $-15.79 * *$ & $19.5^{* *}$ \\
\hline 16 & $\mathrm{P}_{4} \times \mathrm{P}_{5}$ & $-27.76 * *$ & $-7.55 * *$ & $-12.03 * *$ & $-11.32 * *$ & $18.1 * *$ \\
\hline 17 & $\mathrm{P}_{4} \times \mathrm{P}_{6}$ & $-29.10 * *$ & $-10.07^{* *}$ & $-19.70 * *$ & $-17.85^{* *}$ & $18.3^{* *}$ \\
\hline 18 & $\mathrm{P}_{4} \times \mathrm{P}_{7}$ & $-16.47^{*}$ & $-2.11^{\mathrm{H}}$ & $-20.43 * *$ & $-19.96 * *$ & $11.3 * *$ \\
\hline 19 & $\mathrm{P}_{5} \times \mathrm{P}_{6}$ & $-16.56 * *$ & $-10.12 * *$ & $-14.53 * *$ & $-13.27 * *$ & $25.5 * *$ \\
\hline 20 & $\mathrm{P}_{5} \times \mathrm{P}_{7}$ & $-14.49 * \mathrm{H}$ & $-4.48 * *$ & $-6.76 * * \mathrm{H}$ & $-6.55 * * \mathrm{H}$ & $2.99^{\mathrm{L}}$ \\
\hline 21 & $\mathrm{P}_{6} \times \mathrm{P}_{7}$ & $-16.67 * *$ & $-16.21 * *$ & $-18.79 * *$ & $-17.53^{* *}$ & $20.6^{* *}$ \\
\hline L.S.D. ${ }_{0.05}$ & & 0.60 & 1.09 & 0.96 & 0.97 & 0.06 \\
\hline L.S.D. 0.01 & & 0.79 & 1.44 & 1.27 & 1.27 & 0.08 \\
\hline
\end{tabular}

*,* Significant and highly significant at 0.05 and 0.01 probability levels, respectively. 
Table 6. Heterosis versus the mid-parents of the $F_{1 r}$ hybrids for earliness traits

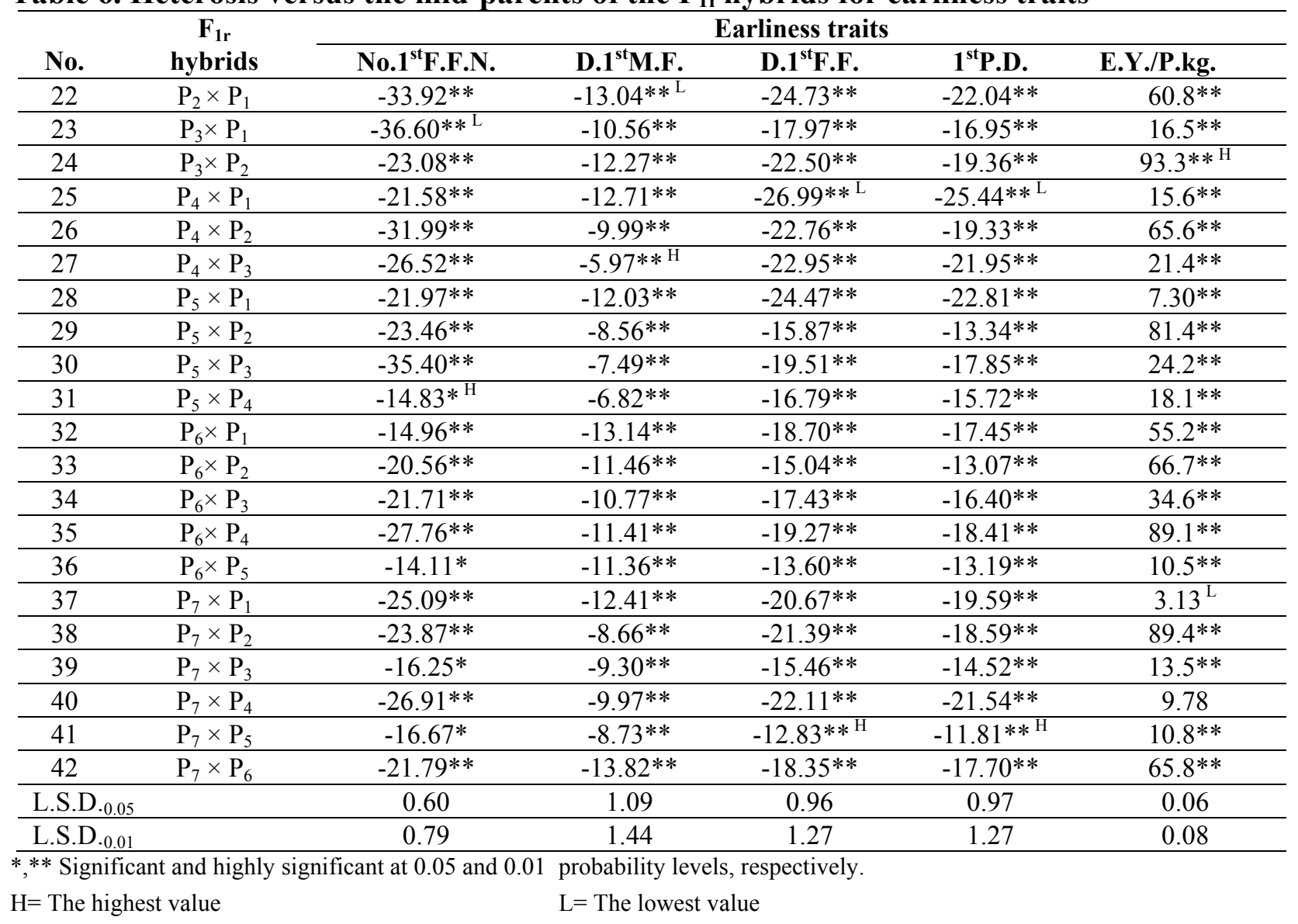

\subsection{Heterosis versus the better parent $\left(\mathbf{H}_{\text {B.P. }} \%\right)$ :-}

Data presented in Tables 7 and 8 gave the heterosis percentage of the $42 \mathrm{~F}_{1,1 \mathrm{r}}$ hybrids relative to the better parent $\left(\mathrm{H}_{\text {B.P. }} \%\right)$ for earliness traits. Most hybrids showed highly significant and desirable negative heterotic values for D. $1^{\text {st } F . F . ~ a n d ~} 1^{\text {st } P . D . ~ A t ~ t h e ~ s a m e ~}$ time, most hybrids showed not significant and desirable negative heterotic values for No. ${ }^{\text {st }}$ F.F.N.

The results showed that heterosis percentages relative to better parent $\left(\mathrm{H}_{\text {B.P. }} \%\right)$ were found to range from -30.73 to $-0.76 \%$ for No. $1^{\text {st } F . F . N . ; ~}-18.52$ to $0.57 \%$ for D. $1^{\text {st }}$ M.F; -26.18 to $-3.35 \%$ for D. $1^{\text {st } F . F ; ~-~}$ 23.80 to $-3.13 \%$ and 2.27 to $74.74 \%$ for E.Y./P.kg. for $\mathrm{F}_{1}$ hybrids. While, the values ranged from -35.40 to $-3.45 \% ;-12.71$ to $-2.67 \% ;-21.63$ to $-2.65 \% ;-20.43$ to -

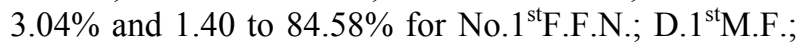
D. ${ }^{\text {st F.F.; }} 1^{\text {st }}$ P.D. and E.Y./P.kg., respectively for $F_{1 r}$ (reciprocal) hybrids.

In general, 4, 16, 18 and $18 \mathrm{~F}_{1}$ hybrids out of the 21 $\mathrm{F}_{1}$ hybrids showed highly significant negative $\left(\mathrm{H}_{\text {B.P. }} \%\right)$ (desirable) values for No. $1^{\text {st }}$ F.F.N.; D. $1^{\text {st }}$ M.F.; D. $1^{\text {st }}$ F.F.; $1{ }^{\text {st }}$ P.D., respectively. In the same time, 4, 14, 19 and 19
$F_{1 \mathrm{r}}$ hybrids out of the $21 \mathrm{~F}_{1 \mathrm{r}}$ hybrids showed similar results for the same traits, respectively.

For E.Y./P.kg., 11 and 12 out of the $21 \mathrm{~F}_{1}$ hybrids and $21 \mathrm{~F}_{1 \mathrm{r}}$ hybrids showed positive and highly significant $\left(\mathrm{H}_{\text {B.P. }} \%\right.$ ) (desirable) estimates, respectively.

Similar results were obtained by El-Adl et al., (1988); Abd El-Hadi and El-Gendy (2004); Abd ElHadi et al., (2005); Obiadalla-Ali (2006); Al-Ballat (2008); Al-Araby (2010); Jahan et al., (2012) and Tamil et al., (2012).

4. Analysis of combining ability variances:

The variance for combining ability of seven varieties and their $42 \mathrm{~F}_{1,1 \mathrm{r}}$ hybrids for earliness traits are shown in Table 9. The results revealed that the mean squares due to crosses were highly significant for all studied traits except for No. $1^{\text {st }}$ F.F.N.

The values of GCA mean squares were higher than those of SCA means for all studied traits. It means that additive genetic variance was more important in the inheritance of these earliness traits, while the reciprocal effect variance were highly significant for E.Y./P.kg. Similar results were obtained by Abd El-Hafez et al., 
(1997); El-Sharkawy (2000); Obiadalla-Ali (2006); Al-

Ballat (2008); Al-Araby, (2010) and Sanin et al., (2014)

Table 7. Heterosis versus the better parent of $F_{1}$ the 21 hybrids for earliness traits

\begin{tabular}{|c|c|c|c|c|c|c|}
\hline \multirow{2}{*}{ No. } & \multirow{2}{*}{$\begin{array}{c}F_{1} \\
\text { hybrids }\end{array}$} & \multicolumn{5}{|c|}{ Earliness traits } \\
\hline & & No. $1^{\text {st }}$ F.F.N. & D.1 ${ }^{\text {st }}$ M.F. & D. ${ }^{\text {st }}$ F.F. & $\mathbf{1}^{\text {st }}$ P.D. & E.Y./P.kg. \\
\hline$T$ & $\mathrm{P}_{1} \times \mathrm{P}_{2}$ & $-24.38 * *$ & $-18.52^{* * L}$ & $-26.18^{* *}$ & $-23.80^{* * 1}$ & $63.31^{* *}$ \\
\hline 2 & $\mathrm{P}_{1} \times \mathrm{P}_{3}$ & $-20.55^{*}$ & $-14.27 * *$ & $-16.41 * *$ & $-15.67 * *$ & 2.81 \\
\hline 3 & $\mathrm{P}_{1} \times \mathrm{P}_{4}$ & $-20.34 *$ & $-15.12 * *$ & $-20.46^{* *}$ & $-18.90 * *$ & 4.55 \\
\hline 4 & $\mathrm{P}_{1} \times \mathrm{P}_{5}$ & $-24.14 * *$ & $-10.51 * *$ & $-17.03 * *$ & $-16.25 * *$ & 3.13 \\
\hline 5 & $\mathrm{P}_{1} \times \mathrm{P}_{6}$ & -9.38 & $-10.28 * *$ & $-17.31 * *$ & $-16.38 * *$ & $62.06 * *$ \\
\hline 6 & $\mathrm{P}_{1} \times \mathrm{P}_{7}$ & -13.74 & $-5.51 * *$ & $-12.49 * *$ & $-11.83 * *$ & 3.22 \\
\hline 7 & $\mathrm{P}_{2} \times \mathrm{P}_{3}$ & -14.38 & $-8.58 * *$ & $-19.63 * *$ & $-17.32 * *$ & $74.49 * *$ \\
\hline 8 & $\mathrm{P}_{2} \times \mathrm{P}_{4}$ & -12.71 & $-10.88 * *$ & $-22.85 * *$ & $-19.26^{* *}$ & $44.23 * *$ \\
\hline 9 & $\mathrm{P}_{2} \times \mathrm{P}_{5}$ & $-20.00 *$ & $-8.63 * *$ & $-21.29 * *$ & $-17.50 * *$ & $53.50 * *$ \\
\hline 10 & $\mathrm{P}_{2} \times \mathrm{P}_{6}$ & $-30.73 * * \mathrm{~L}$ & $-12.04 * *$ & $-13.84 * *$ & $-12.71 * *$ & $74.74 * * \mathrm{H}$ \\
\hline 11 & $\mathrm{P}_{2} \times \mathrm{P}_{7}$ & $-22.90 *$ & $-5.51 * *$ & $-11.98 * *$ & $-9.06 * *$ & $40.14^{* *}$ \\
\hline 12 & $\mathrm{P}_{3} \times \mathrm{P}_{4}$ & $-22.88^{*}$ & $-9.12 * *$ & $-19.63 * *$ & $-16.18 * *$ & $18.64 * *$ \\
\hline 13 & $\mathrm{P}_{3} \times \mathrm{P}_{5}$ & $-22.76^{*}$ & $-8.09 * *$ & $-15.80 * *$ & $-14.73 * *$ & $8.09 *$ \\
\hline 14 & $\overline{\mathrm{P}_{3} \times \mathrm{P}_{6}}$ & $-17.12 *$ & $-5.69 * *$ & $-11.71 * *$ & $-10.88 * *$ & $51.19 * *$ \\
\hline 15 & $\mathrm{P}_{3} \times \mathrm{P}_{7}$ & $-25.19 * *$ & -0.95 & $-11.37 * *$ & $-19.50 * *$ & $7.82 *$ \\
\hline 16 & $\mathrm{P}_{4} \times \mathrm{P}_{5}$ & -19.49 & $-6.36^{* *}$ & $-11.57 * *$ & $-10.76^{* *}$ & $9.84 *$ \\
\hline 17 & $\mathrm{P}_{4} \times \mathrm{P}_{6}$ & -10.17 & -1.20 & $-8.99 * *$ & $-7.59 * *$ & 3.69 \\
\hline 18 & $\mathrm{P}_{4} \times \mathrm{P}_{7}$ & -11.86 & $-0.57^{\mathrm{H}}$ & $-17.97 * *$ & $-17.75^{* *}$ & 2.84 \\
\hline 19 & $\mathrm{P}_{5} \times \mathrm{P}_{6}$ & -6.21 & -2.61 & $-3.69 *$ & $-3.13^{\mathrm{H}}$ & $17.74 * *$ \\
\hline 20 & $\mathrm{P}_{5} \times \mathrm{P}_{7}$ & $-18.62 *$ & -1.71 & $-3.35^{\mathrm{H}}$ & -3.34 & $2.27^{\mathrm{L}}$ \\
\hline 21 & $\mathrm{P}_{6} \times \mathrm{P}_{7}$ & $-0.76^{11}$ & $-6.37 * *$ & $-4.77 *$ & $-4.39^{*}$ & $13.90^{* *}$ \\
\hline L.S.D & & 0.80 & 1.44 & 1.27 & 1.28 & 0.08 \\
\hline L.S.D & & 1.05 & 1.91 & 1.69 & 1.69 & 0.11 \\
\hline
\end{tabular}

*,** Significant and highly significant at 0.05 and 0.01 probability levels, respectively.

$\mathrm{H}=$ The highest value

$\mathrm{L}=$ The lowest value

Table 8. Heterosis versus the better parent of $F_{1 \mathrm{r}}$ the 21 hybrids for earliness traits

\begin{tabular}{|c|c|c|c|c|c|c|}
\hline \multirow[t]{2}{*}{ No. } & \multirow{2}{*}{$\begin{array}{c}\mathbf{F}_{1 \mathbf{r}} \\
\text { hybrids }\end{array}$} & \multicolumn{5}{|c|}{ Earliness traits } \\
\hline & & No.1stF.F.N. & D.1stM.F. & D.1stF.F. & 1stP.D. & E.Y./P.Kg. \\
\hline 22 & $\mathrm{P}_{2} \times \mathrm{P}_{1}$ & $-30.00 * *$ & $-12.71 * * \mathrm{~L}$ & $-21.63 * * \mathrm{~L}$ & $-19.72 * *$ & $57.59 * *$ \\
\hline 23 & $\mathrm{P}_{3} \times \mathrm{P}_{1}$ & $-33.56^{* *}$ & $-8.41 * *$ & $-12.26^{* *}$ & $-11.49^{* *}$ & 4.55 \\
\hline 24 & $\mathrm{P}_{3} \times \mathrm{P}_{2}$ & -14.38 & $-10.51 * *$ & $-20.46^{* *}$ & $-16.62^{* *}$ & $70.40 * *$ \\
\hline 25 & $\mathrm{P}_{4} \times \mathrm{P}_{1}$ & -7.63 & $-8.20 * *$ & $-20.36 * *$ & $-18.90 * *$ & 6.25 \\
\hline 26 & $\frac{4}{P_{4} \times P_{2}}$ & -14.41 & $-5.71 * *$ & $-19.22 * *$ & $-14.92^{* *}$ & $49.41 * *$ \\
\hline 27 & $\mathrm{P}_{4} \times \mathrm{P}_{3}$ & -17.80 & -3.50 & $-21.51 * *$ & $-20.43 * * L$ & $18.31 * *$ \\
\hline 28 & $\mathrm{P}_{5} \times \mathrm{P}_{1}$ & -17.93 & $-8.72 * *$ & $-18.07 * *$ & $-16.61^{* *}$ & 5.97 \\
\hline 29 & $\mathrm{P}_{5} \times \mathrm{P}_{2}$ & -14.48 & $-5.48 * *$ & $-12.49 * *$ & $-9.20 * *$ & $75.58 * *$ \\
\hline 30 & $\mathrm{P}_{5} \times \mathrm{P}_{3}$ & $-35.40 * * \mathrm{~L}$ & $-6.29 * *$ & $-18.45 * *$ & $-16.79 * *$ & $12.72 * *$ \\
\hline 31 & $\mathrm{P}_{5} \times \mathrm{P}_{4}$ & -5.08 & $-5.62 * *$ & $-16.35 * *$ & $-15.19 * *$ & $9.84^{*}$ \\
\hline 32 & $\mathrm{P}_{6} \times \mathrm{P}_{1}$ & -9.38 & $-9.44 * *$ & $-15.78 * *$ & $-14.85 * *$ & $47.36 * *$ \\
\hline 33 & $\mathrm{P}_{6} \times \mathrm{P}_{2}$ & $-20.11 * *$ & $-7.32 * *$ & $-8.23 * *$ & $-7.58 * *$ & $61.41 * *$ \\
\hline 34 & $\mathrm{P}_{6} \times \mathrm{P}_{3}$ & -12.33 & -4.64 & $-8.29 * *$ & $-7.92 * *$ & $15.44 * *$ \\
\hline 35 & $\mathrm{P}_{6} \times \mathrm{P}_{4}$ & -8.47 & $-2.67^{\mathrm{H}}$ & $-8.51 * *$ & $-8.23 * *$ & $65.75 * *$ \\
\hline 36 & $\mathrm{P}_{6} \times \mathrm{P}_{5}$ & $-3.45^{\mathrm{H}}$ & -3.95 & $-2.65^{\mathrm{H}}$ & $-3.04^{\mathrm{H}}$ & 3.69 \\
\hline 37 & $\mathrm{P}_{7} \times \mathrm{P}_{1}$ & -16.79 & $-6.37 * *$ & $-10.56^{* *}$ & $-9.92 * *$ & 2.56 \\
\hline 38 & $\mathrm{P}_{7} \times \mathrm{P}_{2}$ & -9.92 & -2.76 & $-15.13 * *$ & $-11.64^{* *}$ & $84.58 * * \mathrm{H}$ \\
\hline 39 & $\mathrm{P}_{7} \times \mathrm{P}_{3}$ & -11.45 & $-5.42 *$ & $-11.17 * *$ & $-10.40 * *$ & 2.43 \\
\hline 40 & $\mathrm{P}_{7} \times \mathrm{P}_{4}$ & $-22.88 *$ & $-8.56 * *$ & $-19.70 * *$ & $-19.37 * *$ & $1.40^{\mathrm{L}}$ \\
\hline 41 & $\mathrm{P}_{7} \times \mathrm{P}_{5}$ & -12.21 & $-6.08 * *$ & $-9.64 * *$ & $-8.78 * *$ & $10.02 *$ \\
\hline 42 & $\mathrm{P}_{7} \times \mathrm{P}_{6}$ & -6.87 & -3.71 & $-4.26^{*}$ & $-4.58 *$ & $56.56 * *$ \\
\hline
\end{tabular}




\begin{tabular}{lccccc}
\hline L.S.D. $\cdot 0.05$ & 0.80 & 1.44 & 1.27 & 1.28 & 0.08 \\
\hline L.S.D. $\cdot 0.01$ & 1.05 & 1.91 & 1.69 & 1.69 & 0.11 \\
\hline *** Significant and highly significant at 0.05 and 0.01 & probability levels, respectively. \\
H= The highest value & \multicolumn{2}{c}{ L= The lowest value }
\end{tabular}

Table 9. Analysis of combining abilities and mean squares of all $F_{1}$ hybrids $\left(F_{1,1 r}\right.$ hybrids) for earliness traits

\begin{tabular}{|c|c|c|c|c|c|c|}
\hline \multirow[b]{2}{*}{ S.V. } & \multirow[t]{2}{*}{ d.f. } & \multicolumn{5}{|c|}{ Earliness traits } \\
\hline & & No.1 ${ }^{\text {st }}$ F.F.N. & D.1 ${ }^{\text {st }}$ M.F. & D. $1^{\text {st }} \mathbf{F} . \mathbf{F}$ & $\mathbf{1}^{\text {st }}$ P.D. & E.Y./P.kg. \\
\hline Reps. & 2 & 0.002 & 0.149 & 0.005 & $6.740 * *$ & 0.012 \\
\hline Crosses & 41 & 0.274 & $3.913 * *$ & $9.870 * *$ & $9.568 * *$ & $0.140 * *$ \\
\hline G.C.A. & 6 & $1.536 * *$ & $10.825^{* *}$ & $39.307 * *$ & $39.887 * *$ & $0.891 * *$ \\
\hline S.C.A. & 14 & $0.463 * *$ & $6.364 * *$ & $18.282 * *$ & $17.759 * *$ & $0.203 * *$ \\
\hline R.E. & 21 & 0.072 & 1.278 & 0.989 & 0.923 & $0.071 * *$ \\
\hline Error & 82 & 0.245 & 0.8 & 0.626 & 0.631 & 0.003 \\
\hline G.C.A./ S.C.A. & - & 0.423 & 0.129 & 0.156 & 0.164 & 0.317 \\
\hline
\end{tabular}

*** Significant at 0.05 and 0.01 levels of probability, respectively

\section{Genetic parameters and heritability:}

The relative magnitudes of genetic parameters and heritability for earliness traits were estimated and the obtained results are shown in Table 10.

It appeared that, both additive $\left(\delta^{2} \mathrm{~A}\right)$, non-additive genetic variances including dominance $\left(\delta^{2} \mathrm{D}\right)$ genetic variance were contributed in the inheritance of number of first female flowering node (No. $1^{\text {st }}$ F.F.N.); date of first male flower (D. $1^{\text {st }}$ M.F.); data of first female flower (D. $1^{\text {st }}$ F.F. $)$; first picking date $\left(1^{\text {st P.D. }}\right)$ and early yield per plant (E.Y./P.kg.).

The dominance $\left(\delta^{2} \mathrm{D}\right)$ genetic variance was larger than their corresponding values of additive genetic variance $\left(\delta^{2} \mathrm{~A}\right)$ for No. $1^{\mathrm{st}}$ F.F.N trait. This suggests that dominance genetic variance played the major role in the genetic expression of this trait. Although, the magnitudes of $\delta^{2} \mathrm{~A}$, were also smaller than $\delta^{2} \mathrm{D}$ for D. $1^{\text {st }}$ M.F.; D. $1^{\text {st F.F.; }} \quad 1^{\text {st P.D. }}$ and E.Y./P.kg. This suggests that additive genetic variance played was presented role in the genetic expression of these traits. Furthermore, the reciprocal effect variances $\left(\delta^{2} \mathrm{r}\right)$ were positive for all studied earliness traits except No. $1^{\text {st }}$ F.F.N trait, indicating that cytoplasmic factor played an important role in the expression of these traits in addition to nuclear genes.

Also, the estimates of heritability in broad sense $\left(\mathrm{h}^{2}{ }_{\mathrm{b}}\right.$ $\%)$ were larger than their corresponding estimates in narrow sense $\left(\mathrm{h}_{\mathrm{n}}^{2} \%\right)$ for all studied earliness traits. These values were $57.242 ; 85.237 ; 95.975 ; 95.851$ and $98.863 \%$ in broad sense and $34.554 ; 19.486 ; 26.988$; 27.600 and $40.909 \%$ in narrow sense for No. $1^{\text {st }}$ F.F.N; D. $1^{\text {st M.F.; }} \quad$ D. $1^{\text {st }}$ F.F.; $\quad 1^{\text {st P.D. }} \quad$ and $\quad$ E.Y./P.kg., respectively.

In this respect, many authors, such as Abd El-Hadi and El-Gendy (2004); Mishra et al. (2007); Al-Ballat (2008); Al-Araby, (2010) and Sanin et al., (2014) reported that both additive and non-additive genetic variances contributed in the inheritance of earliness traits.

6. General combining ability effects $\left(g_{i}\right)$ for the parents:

The general combining ability effects $\left(\mathrm{g}_{\mathrm{i}}\right)$ of the seven parental varieties for earliness traits are shown in Table 11.

The results revealed that the GCA effects $\left(g_{i}\right)$ showed desirable negative and highly significant values to the parental variety $\mathrm{P}_{4}$ for No. $1^{\text {st }}$ F.F.N.; D. $1^{\text {st } M . F . ; ~}$ D. $1^{\text {st }}$ F.F. and $1^{\text {st }}$ P.D. The results revealed that the GCA effects $\left(g_{i}\right)$ showed desirable negative and highly significant values to the parent $\mathrm{P}_{7}$ for D. $1^{\text {st } M . F}$.; D. $1^{\text {st }}$ F.F. and $1^{\text {st }}$ P.D. Meanwhile, the GCA effects were found to be highly significant and positive (undesirable) for the parent $\mathrm{P}_{6}$ for all earliness traits except for E.Y./P.kg. which was desirable.

These results indicated that the parents $\mathrm{P}_{4}$ and $\mathrm{P}_{7}$ seemed to be the best combiners for earliness traits No. $1^{\text {st }}$ F.F.N.; D. $1^{\text {st } M . F . ; ~ D . ~} 1^{\text {st }}$ F.F. and $11^{\text {st }}$ P.D. The two parents No. $\mathrm{P}_{2}$ and $\mathrm{P}_{6}$ were the best combiners for E.Y./P.kg. Similar results were obtained by ElSharkawy (2000); Al-Ballat (2008); Al-Araby, (2010) and Sanin et al., (2014).

\section{Specific combining ability effects $\left(s_{\mathrm{ij}}\right)$ :}

Estimates of specific combining ability effects $\left(\mathrm{s}_{\mathrm{ij}}\right)$ of the $42 F_{1, \text { lr }}$ hybrids for earliness traits are presented in Tables 12 and 13.

The $\mathrm{F}_{1}$ hybrids $\mathrm{P}_{3} \times \mathrm{P}_{5}$ and $\mathrm{P}_{4} \times \mathrm{P}_{6}$ did not show significant negative (desirable) SCA effects for No. $1^{\text {st }}$ F.F.N. While, the $\mathrm{F}_{1 \mathrm{r}}$ (reciprocal) hybrids $\mathrm{P}_{6} \times \mathrm{P}_{2}$ and $\mathrm{P}_{7} \times \mathrm{P}_{2}$ show did not significant negative (desirable) SCA effects for the same trait. At the same time, the $\mathrm{F}_{1 \mathrm{r}}$ (reciprocal) hybrid $\mathrm{P}_{7} \times \mathrm{P}_{2}$ gave the highest negative value $(-0.321)$ for the same trait. 
For D. $1^{\text {st }}$ M.F. the $\mathrm{F}_{1}$ hybrids $\mathrm{P}_{1} \times \mathrm{P}_{4}$ and $\mathrm{P}_{6} \times \mathrm{P}_{7}$ gave the highest desirable negative value $(-0.321)$, while, the $\mathrm{F}_{1 \mathrm{r}}$ (reciprocal) hybrid $\mathrm{P}_{4} \times \mathrm{P}_{1}$ gave the highest negative value $(-1.667)$ for the same trait.

Table 10. The relative magnitudes of different genetic parameters and heritability for earliness traits

\begin{tabular}{|c|c|c|c|c|c|}
\hline \multirow{2}{*}{$\begin{array}{l}\text { Genetic parameters } \\
\text { and heritability }\end{array}$} & \multicolumn{5}{|c|}{ Earliness traits } \\
\hline & No.1 ${ }^{\text {st }}$ F.F.N. & D.1 ${ }^{\text {st }}$ M.F. & D.1 ${ }^{\text {st }}$ F.F. & $\mathbf{1}^{\text {st }}$ P.D. & E.Y./P.kg. \\
\hline$\delta^{2} \mathrm{~A}$ & 0.198 & 1.056 & 4.068 & 4.198 & 0.108 \\
\hline$\delta^{2} \mathrm{D}$ & 0.130 & 3.324 & 10.550 & 10.235 & 0.119 \\
\hline$\delta^{2} \mathrm{r}$ & -0.086 & 0.239 & 0.181 & 0.146 & 0.034 \\
\hline$\delta^{2} \mathrm{E}$ & 0.245 & 0.800 & 0.626 & 0.631 & 0.003 \\
\hline$h^{2} b \%$ & 57.242 & 85.237 & 95.975 & 95.851 & 98.863 \\
\hline $\mathrm{h}^{2} \mathrm{n} \%$ & 34.554 & 19.486 & 26.988 & 27.600 & 40.909 \\
\hline
\end{tabular}

Note: Negative values were considered equal to zero during the calculation of heritability in broad and narrow senses

Table 11. General combining ability effects (gi) of the seven parents for earliness traits

\begin{tabular}{|c|c|c|c|c|c|}
\hline \multirow[b]{2}{*}{ Parents } & \multicolumn{5}{|c|}{ Earliness traits } \\
\hline & No.1 ${ }^{\text {st }}$ F.F.N. & D.1 ${ }^{\text {st }}$ M.F. & D.1 ${ }^{\text {st F.F. }}$ & $1^{\text {st }}$ P.D. & E.Y./P.kg. \\
\hline $\mathrm{P}_{1}$ & 0.08 & -0.299 & $0.418^{*}$ & $0.449 *$ & $-0.061 * *$ \\
\hline $\mathrm{P}_{2}$ & 0.155 & 0.081 & $-0.415^{*}$ & 0.139 & $0.510 * *$ \\
\hline $\mathrm{P}_{3}$ & -0.136 & 0.153 & $-0.415^{*}$ & $-0.527 * *$ & $-0.132 * *$ \\
\hline $\mathrm{P}_{4}$ & $-0.509 * *$ & $-0.727 * *$ & $-1.556^{* *}$ & $-1.693 * *$ & $-0.132 * *$ \\
\hline $\mathrm{P}_{5}$ & 0.026 & 0.010 & -0.129 & -0.074 & $-0.204 * *$ \\
\hline $\mathrm{P}_{6}$ & $0.562 * *$ & $1.748 * *$ & $3.466^{* *}$ & $3.329 * *$ & $0.153 * *$ \\
\hline $\mathrm{P}_{7}$ & -0.179 & $-0.966 * *$ & $-1.367 * *$ & $-1.622 * *$ & $-0.132 * *$ \\
\hline L.S.D(gi) $\cdot_{0.05}$ & 0.242 & 0.438 & 0.387 & 0.389 & 0.026 \\
\hline L.S.D(gi).0.01 & 0.319 & 0.577 & 0.510 & 0.513 & 0.035 \\
\hline
\end{tabular}

*,**Significant and highly significant at 0.05 and 0.01 probability levels, respectively.

Table 12. Specific combining ability effects $\left(s_{i j}\right)$ of the $21 F_{1}$ hybrids for earliness traits

\begin{tabular}{|c|c|c|c|c|c|}
\hline \multirow[b]{2}{*}{$F_{1}$ hybrids } & \multicolumn{5}{|c|}{ Earliness traits } \\
\hline & No.1 ${ }^{\text {st }}$ F.F.N. & D.1 $1^{\text {st }}$ M.F. & D.1 ${ }^{\text {st }}$ F.F & $\mathbf{1}^{\text {st }}$ P.D. & E.Y./P.kg. \\
\hline $\mathrm{P}_{1} \times \mathrm{P}_{2}$ & -0.370 & -1.224 & $-2.228 * *$ & $-2.068 * *$ & $0.204 * *$ \\
\hline $\mathrm{P}_{1} \times \mathrm{P}_{3}$ & -0.346 & -0.796 & -0.228 & -0.235 & $-0.153 * *$ \\
\hline $\mathrm{P}_{1} \times \mathrm{P}_{4}$ & -0.164 & $-1.748 *$ & $-2.418 * *$ & $-2.235 * *$ & $-0.153 * *$ \\
\hline $\mathrm{P}_{1} \times \mathrm{P}_{5}$ & -0.294 & -0.986 & $-2.347 * *$ & $-2.687 * *$ & -0.081 \\
\hline $\mathrm{P}_{1} \times \mathrm{P}_{6}$ & 0.171 & -0.224 & -0.942 & -0.759 & $0.561 * *$ \\
\hline $\mathrm{P}_{1} \times \mathrm{P}_{7}$ & -0.176 & -0.510 & -1.109 & -1.139 & $-0.153 * *$ \\
\hline $\mathrm{P}_{2} \times \mathrm{P}_{3}$ & 0.184 & -1.010 & -1.228 & $-1.425^{*}$ & $0.275 * *$ \\
\hline $\mathrm{P}_{2} \times \mathrm{P}_{4}$ & -0.206 & -0.796 & $-1.585^{*}$ & $-1.425^{*}$ & $0.275 * *$ \\
\hline $\mathrm{P}_{2} \times \mathrm{P}_{5}$ & -0.156 & -0.534 & $-1.347^{*}$ & -1.044 & $0.346 * *$ \\
\hline $\mathrm{P}_{2} \times \mathrm{P}_{6}$ & -0.248 & -0.772 & -0.276 & -0.449 & -0.010 \\
\hline $\mathrm{P}_{2} \times \mathrm{P}_{7}$ & -0.293 & -0.058 & -0.942 & -0.997 & $0.275 * *$ \\
\hline $\mathrm{P}_{3} \times \mathrm{P}_{4}$ & -0.187 & -0.367 & -0.918 & -1.092 & -0.082 \\
\hline $\mathrm{P}_{3} \times \mathrm{P}_{5}$ & -0.455 & -0.272 & $-1.847 * *$ & $-1.378^{*}$ & -0.010 \\
\hline $\mathrm{P}_{3} \times \mathrm{P}_{6}$ & -0.256 & -0.510 & $-1.609 *$ & $-1.449 *$ & $0.132 * *$ \\
\hline $\mathrm{P}_{3} \times \mathrm{P}_{7}$ & -0.091 & 0.037 & -0.109 & -0.163 & -0.081 \\
\hline $\mathrm{P}_{4} \times \mathrm{P}_{5}$ & -0.017 & -0.224 & 0.629 & 0.456 & -0.010 \\
\hline $\mathrm{P}_{4} \times \mathrm{P}_{6}$ & -0.459 & -0.296 & -1.299 & -1.116 & $0.132 * *$ \\
\hline $\mathrm{P}_{4} \times \mathrm{P}_{7}$ & -0.066 & 0.418 & -1.299 & $-1.663^{*}$ & -0.081 \\
\hline $\mathrm{P}_{5} \times \mathrm{P}_{6}$ & 0.025 & -0.534 & -0.228 & -0.568 & $-0.295 * *$ \\
\hline $\mathrm{P}_{5} \times \mathrm{P}_{7}$ & 0.052 & 0.014 & 0.772 & 1.051 & -0.010 \\
\hline $\mathrm{P}_{6} \times \mathrm{P}_{7}$ & -0.163 & $-2.391 * *$ & $-1.823 * *$ & $-1.687^{*}$ & $0.132 * *$ \\
\hline L.S.D. $\left(\mathrm{s}_{\mathrm{ij}}\right)_{0.05}$ & 0.840 & 1.517 & 1.342 & 1.348 & 0.092 \\
\hline
\end{tabular}




\begin{tabular}{|c|c|c|c|c|c|}
\hline L.S.D. $\left(\mathrm{s}_{\mathrm{ij}}\right)_{0.01}$ & 1.107 & 2.000 & 1.770 & 1.777 & 0.122 \\
\hline \multicolumn{6}{|c|}{$\begin{array}{l}*, * \text { Significant and highly significant at } 0.05 \text { and } 0.01 \text { probability levels, respectively } \\
\text { Table 13. Specific combining ability effects }\left(\mathbf{r}_{\mathbf{i j}}\right) \text { of the } \mathbf{2 1} \mathbf{F}_{\mathbf{1 r}} \text { hybrids for earliness traits }\end{array}$} \\
\hline \multirow[t]{2}{*}{$\mathrm{F}_{1 \mathrm{r}}$ hybrids } & \multicolumn{5}{|c|}{$\begin{array}{l}\text { Earliness traits } \\
\end{array}$} \\
\hline & No.1 ${ }^{\text {st }}$ F.F.N. & D.1 ${ }^{\text {st }}$ M.F. & D.1 ${ }^{\text {st }} \mathbf{F} . \mathbf{F}$. & $\mathbf{1}^{\text {st }} \mathbf{P . D .}$ & E.Y./P.kg. \\
\hline $\mathrm{P}_{2} \times \mathrm{P}_{1}$ & 0.121 & -1.00 & -1.00 & -0.833 & 0.001 \\
\hline $\mathrm{P}_{3} \times \mathrm{P}_{1}$ & 0.306 & -1.167 & -0.667 & -0.667 & 0.001 \\
\hline $\mathrm{P}_{3} \times \mathrm{P}_{2}$ & 0.005 & 0.667 & 0.167 & -0.167 & 0.001 \\
\hline $\mathrm{P}_{4} \times \mathrm{P}_{1}$ & -0.255 & $-1.667 * *$ & 0.001 & -0.167 & 0.001 \\
\hline $\mathrm{P}_{4} \times \mathrm{P}_{2}$ & 0.041 & -1.00 & -0.667 & -1.00 & 0.001 \\
\hline $\mathrm{P}_{4} \times \mathrm{P}_{3}$ & -0.075 & -0.833 & 0.667 & 0.667 & 0.001 \\
\hline $\mathrm{P}_{5} \times \mathrm{P}_{1}$ & -0.121 & -0.167 & 0.167 & 0.0001 & 0.001 \\
\hline $\mathrm{P}_{5} \times \mathrm{P}_{2}$ & -0.128 & -0.667 & $-1.667 * *$ & $-1.667 * *$ & 0.001 \\
\hline $\mathrm{P}_{5} \times \mathrm{P}_{3}$ & 0.303 & -0.333 & 0.500 & 0.333 & 0.001 \\
\hline $\mathrm{P}_{5} \times \mathrm{P}_{4}$ & -0.255 & -0.500 & 0.833 & 1.000 & 0.001 \\
\hline $\mathrm{P}_{6} \times \mathrm{P}_{1}$ & -0.040 & -0.333 & -0.167 & -0.333 & 0.001 \\
\hline $\mathrm{P}_{6} \times \mathrm{P}_{2}$ & -0.318 & -0.833 & $-1.333 *$ & -1.00 & 0.001 \\
\hline $\mathrm{P}_{6} \times \mathrm{P}_{3}$ & -0.108 & -0.167 & -0.667 & -0.667 & $0.500 * *$ \\
\hline $\mathrm{P}_{6} \times \mathrm{P}_{4}$ & -0.031 & 0.167 & -0.167 & 0.167 & $-0.500 * *$ \\
\hline $\mathrm{P}_{6} \times \mathrm{P}_{5}$ & -0.066 & 0.333 & 0.001 & 0.001 & 0.001 \\
\hline $\mathrm{P}_{7} \times \mathrm{P}_{1}$ & 0.080 & 0.001 & -0.500 & -0.333 & 0.001 \\
\hline $\mathrm{P}_{7} \times \mathrm{P}_{2}$ & -0.321 & -0.167 & 0.500 & 0.500 & 0.001 \\
\hline $\mathrm{P}_{7} \times \mathrm{P}_{3}$ & -0.288 & 0.667 & 0.001 & -0.333 & 0.001 \\
\hline $\mathrm{P}_{7} \times \mathrm{P}_{4}$ & 0.216 & $1.833 * *$ & 0.333 & 0.333 & 0.001 \\
\hline $\mathrm{P}_{7} \times \mathrm{P}_{5}$ & 0.055 & 0.500 & $1.167 *$ & 1.000 & 0.001 \\
\hline $\mathrm{P}_{7} \times \mathrm{P}_{6}$ & 0.135 & -0.500 & -0.167 & 0.001 & $-0.500 * *$ \\
\hline L.S.D. $\left(\mathrm{r}_{\mathrm{ij}}\right)_{0.05}$ & 0.693 & 1.252 & 1.107 & 1.112 & 0.076 \\
\hline L.S.D. $\left(\mathrm{r}_{\mathrm{ij}}\right)_{0.01}$ & 0.913 & 1.650 & 1.460 & 1.466 & 0.101 \\
\hline
\end{tabular}

*,** Significant and highly significant at 0.05 and 0.01 probability levels, respectively .

For D. ${ }^{\text {st }}$ F.F. the $\mathrm{F}_{1}$ hybrids $\mathrm{P}_{1} \times \mathrm{P}_{4}$ and $\mathrm{P}_{1} \times \mathrm{P}_{5}$ gave the highest decimal negative (desirable) values -2.418 and -2.347 , respectively. While, the $\mathrm{F}_{1 \mathrm{r}}$ (reciprocal) hybrid $\mathrm{P}_{5} \times \mathrm{P}_{2}$ gave the highest negative value -1.667 for the same trait.

For $1^{\text {st }}$ P.D. the $\mathrm{F}_{1}$ hybrids $\mathrm{P}_{1} \times \mathrm{P}_{4}$ and $\mathrm{P}_{1} \times \mathrm{P}_{5}$ gave the highest negative (desirable) significant values 2.235 and -2.687 , respectively. While, the $F_{1 \mathrm{r}}$ (reciprocal) hybrid $\mathrm{P}_{5} \times \mathrm{P}_{2}$ gave the highest negative significant value -1.667 for the same trait.

For E.Y./P.kg. the $\mathrm{F}_{1}$ hybrids $\mathrm{P}_{1} \times \mathrm{P}_{6}$ and $\mathrm{P}_{2} \times \mathrm{P}_{5}$ gave the highest positive desirable significant values 0.561 and 0.346 , respectively. While, the $F_{1 \mathrm{r}}$ (reciprocal) hybrid $\mathrm{P}_{6} \times \mathrm{P}_{3}$ gave the highest positive desirable highly significant value 0.500 for the same trait.

Similar results were obtained by Abd El-Hafez et al., (1997); El-Sharkawy (2000); Abd El-Hadi et al., (2005); Obiadalla-Ali (2006); Al-Ballat (2008); AlAraby, (2010) and Sanin et al., (2014).

\section{REFERENCES}

Abd El-Hadi, A. H.; A. M. El-Adl; M. S. Hamada and M. A. Abdein (2005). Manifestation of heterosis and genetic parameters associated with it for some vegetative and earliness traits in squash. J. Agric. Sci., Mansoura Univ., 30(3): 1363-1379.

Abd El-Hadi, A. H. and Soher E. A. El-Gendy (2004). Effect of genotypes by locations interaction on economical traits of squash. J. Agric. Sci., Mansoura Univ., 29(10): 55675587.

Abd El-Hafez, A. A.; S. F. El-Sayyed and A. A. Gharib (1997). Genetic analysis of cucumber yield and its components by diallel crossing. Egypt. J. Hort. 24(2): 141-159.

Abd El-Maksoud, M. M.; A. M. El-Adl; M. S. Hamada and M. S. Sadek (2003). Inheritance of some economical traits in squash (Cucurbita pepo, L.). J. Agric. Sci. Mansoura Univ., 28(6): 4463-4474.

Al-Araby, A. A. (2010). Estimation of heterosis, combining ability and heritability in intervarietal crosses of summer 
squash (Cucurbita pepo L.) .Ph. D. Thesis, Fac. of Agric., Tanta Univ. Egypt.

Al-Ballat, I. A. (2008). Breeding studies on summer squash crop (Cucurbita pepo L.). M.Sc. Thesis, Fac. of Agric., Tanta Univ. Egypt.

Cockerham, C. C. (1963). Estimation of genetic variances. Statistical Genetics and Plant Breeding. NAS-NRC, 982, pp.53-68.

El-Adl, A. M; Z. A. Kosba; T. M. El-Gazar and M. M. ElMaksoud (1988). Manifestation of heterosis and types of genetic effects associated with it for some quantitative traits in squash (Cucurbita pepo, L.) I. Magnitudes of heterosis and inbreeding depression. J. Agric. Sci. Mansoura Univ., 13(3): 1287-1295.

El-Gendy, Soher E. A. (1999). Estimates of genetic parameters in some squash hybrids through two mating designs. Ph. D. Thesis, Fac. of Agric. Mansoura Univ., Egypt.

El-Sharkawy, Gehan A. M. (2000). An analytical study for the genetic behavior of some important characters of summer squash (Cucurbita pepo,L.) using a diallel cross system among seven inbred lines of "Eskandrani" cultivar. M. Sc. Thesis, Fac. of Agric., Alex. Univ., Egypt.

Griffing, B. (1956). Concept of general and specific combining ability in relation to diallel crosses system. Aust. J. Biol. Sci., 9: 463-493.

Jahan, T. A.; A. K. M. Islam; M. G. Rasul; M. A. K. Mian and M. M. Haque (2012). Heterosis of qualitative and quantitative characters in sweet gourd (Cucurbita moschata Duch.ex Poir). African Journal of Food, Agriculture, Nutrition and Development. 12 (3): 61866199.
Matzinger, D. F. and O. Kempthorne (1956). The modified diallel Table with partial inbreeding and interactions with environment., Gene., 41(1): 822-833.

Mishra, G.; J. R. Yadav; N. S. Parihar; J. K. Yadav; S. Kumar and Y. Alok (2007). Study on heritability and genetic advance in cucumber (Cucumis sativus, L.). Progressive Research 2:187.

Obiadalla-Ali, H. A. (2006). Heterosis and nature of gene action for earliness and yield components in summer squash (Cucurbita pepo, L.). Assuit J. of Agric. Sci., 37:123-135.

Sadek, Mariam S. S. (2003). Inheritance of some economical traits in squash (Cucurbita pepo, L.). M. Sc. Thesis, Fac. of Agric., Mansoura Univ. Egypt.

Sanin, O. G.; L. V. B. Burbano; G. A. O. Narvaez; M. P. V. Restrepo; D. B. Garcia and F. A. V. Cabrera (2014). Inbreeding and gene action in butternut squash (Cucurbita moschata) seed starch content. Rev. Fac. Nal. Agr. Medellin, 67(1): 7169-7175.

Steel, G. D. and H. Torrie (1960). Principles and procedures of statistics. Mc. raw. Hill Book Company, INC, New York, PP. 431.

Tamil, N. A.; P. Jansirani; L. Pugalendhi and A. Nirmalakumari (2012). Per se performance of genotypes and correlation analysis in Pumpkin (Cucurbita moschata Duch.ex Poir). Electronic Journal of Plant Breeding, 3(4): 987- 994

Zuradzka, I. (1988). Heredity of the number of female flowers in the node and its influence upon yielding of cucumber fruits. Proceeding of the Europian meeting on cucurbit genetics and breeding. Mysore J. Agric. Sci., 15(3): 452455. 


\section{الملغص العري}

\section{قوة الهجن ومعالل التوريث والقدرة علي التآل لبهض صفل التبكيرفي قرع الكوسة}

على ماهر العل، لثُرف جسين عبد الهادي، حورية محمد فتهي ومحمد عبد الحميد عابنين

لظهرت النت ائج أن Courgette Orelia (الأب الرابـ ع) و Gapla (الأب الساعع) يعتبرلن أفضل مصدر لصفلت رقم

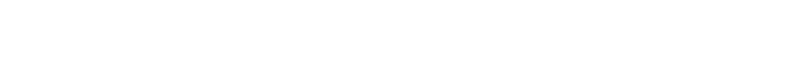

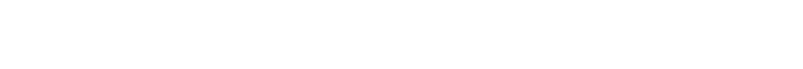
الأيلم حقى أول جمعة، بينما كلن Zucca Patisson custard

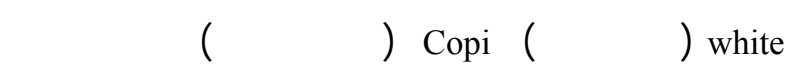

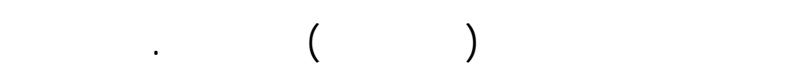
النتائج وجود لختلافلت كبيرة بين هجن الجيل الأول والأول العكسي مع عدم تميز هجين معين بذاته الكل صفلت التبكير المدروسة، ولكن معظم الترلكي ـب الوراثي ـة للجي لـ الألول

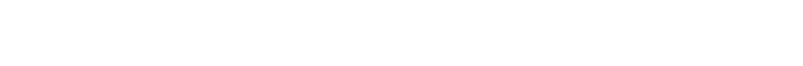

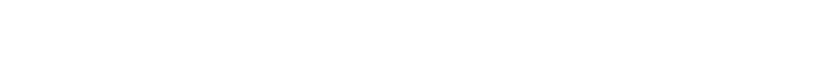
الدرلسة.

أظهرت النتائج تعظم قيم كل من القدرة العلمة على التآف (GCA) والقدرة الخاص هة على الت آلف (SCA).

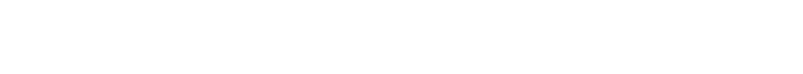
صفلت التبكيرالتي تمت درلستها للجيل الأول الهجين، بينما

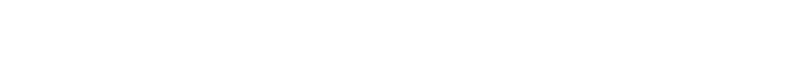

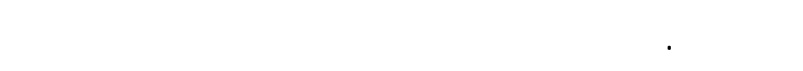
والضيق لجمبع الصفلت مطل الدرلسة وقيم معلل التوريث

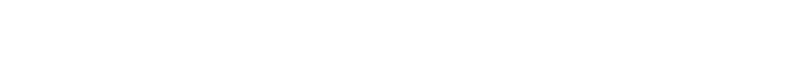
الصفلت مطل الدرلسة.
لجريت هذه الدرلسة في مزرعة قها البحثية التابعة القم

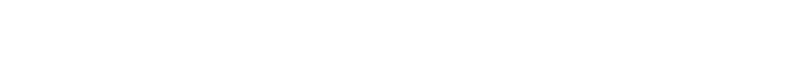

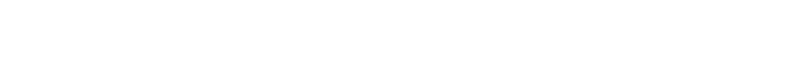

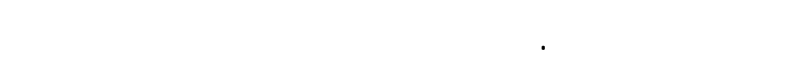
قوة الهجين قيلسا من متوسط الآباء وأفضل الآباء، والقدرة العلمة والخاصة علي التآف وطبيعة فعل الجين، ومعلم لـ لـ لمالِ التوريث في مدd الولسع والضيق لبعض صـ فلت التبكي -ر

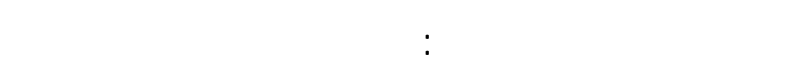
مؤنثة، عدد الأيلم حتق تفتح أول زهرة مذكرة، عدد الأي لم الم

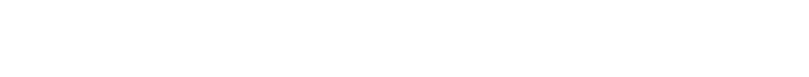
المحصول المبكر(بالكيلوجراd) لكل نبلت.

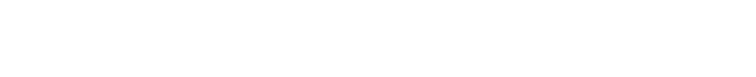

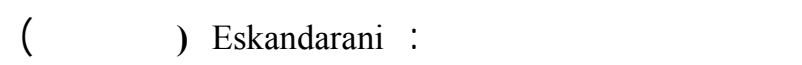
All Green Zucca Patisson custard white Sakiz (الأب الثال)، Bush

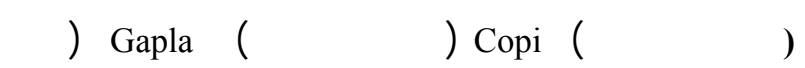
(السابع) (البع) قم التهجين الدوري الكلمل في التجاهين بين جمبع الأباء المستخدة في الدرلسة ونتج عنه r اع هجينا نص فها (Y)

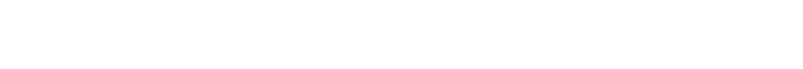
ذات قاعلت كلملة العثوائية من ثلاث مكررات. 